\title{
Automatic Cell Detection and Segmentation from H\&E Stained Pathology Slides using Colorspace Decorrelation Stretching
}

\author{
Mohammad Peikari ${ }^{1}$, Anne L. Martel ${ }^{1,2}$ \\ ${ }^{1}$ Medical Biophysics, University of Toronto, Toronto, Canada; \\ ${ }^{2}$ Physical Sciences, Sunnybrook Research Institute, Toronto, Canada
}

\begin{abstract}
Purpose: Automatic cell segmentation plays an important role in reliable diagnosis and prognosis of patients.

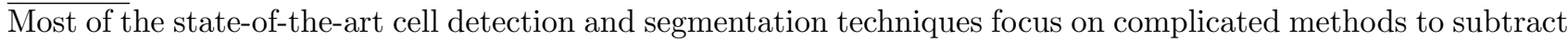
foreground cells from the background. In this study, we introduce a preprocessing method which leads to a better detection and segmentation results compared to a well-known state-of-the-art work. Method: We transform the original red-green-blue (RGB) space into a new space defined by the top eigenvectors of the RGB space. Stretching is done by manipulating the contrast of each pixel value to equalize the color variances. New pixel values are then inverse transformed to the original RGB space. This altered RGB image is then used to segment cells. Result: The validation of our method with a well-known state-of-the-art technique revealed a statistically significant improvement on an identical validation set. We achieved a mean F1-score of 0.901. Conclusion: Preprocessing steps to decorrelate colorspaces may improve cell segmentation performances.
\end{abstract}

Keywords: digital pathology, cell segmentation, decorrelation stretch, automated image analysis.

\section{INTRODUCTION}

Quantitative analysis of digital pathology slides plays an important role in diagnosis and prognosis of patients. Cell detection and segmentation is one of the many tasks necessary for reliable investigations in many scientific and clinical applications including cancer detection and grading. Therefore, reproducible automated detection and segmentation of cells in pathology slides is essential. Studies related to cell segmentation usually focus on complicated methods to robustly detect nuclei from background or separate overlapping ones (reader is referred to the work done by Irshad et. $a l^{1}$ for a review of the recently proposed methods). However, there have been only a few studies that focus on the preprocessing aspects of pathology images which lead to a better and more reliable cell segmentation.

In this study, we introduce a preprocessing method based on decorrelation and stretching of the colorspace from pathology slides which enhances the segmentation performance. We applied our segmentation technique on a publicly available groundtruth dataset provided $\mathrm{in}^{2}$ and compared its performance with their presented results.

\section{PREVIOUS WORKS}

Nuclei detection and segmentation from H\&E stained images is now a well established research area. There have been many methods proposed in the literature that use one or a combination of different image processing techniques including thresholding, region growing, watershed, morphological operations, graph cuts, active contours, probabilistic models, and $K$-means clustering. ${ }^{1}$ In this paper, we consider two of the most recent and well-known approaches for nuclei detection and segmentation present in the literature. ${ }^{2,3}$

The approach taken by Al-Kofahi et al. ${ }^{3}$ was to have an initial stage of image binarization through graph cuts followed by applying a multi-scale Laplacian of Gaussian (LoG) to identify and separate connected blobs

Further author information: (Send correspondence to Mohammad Peikari)

M. Peikari: E-mail: mpeikari@sri.utoronto.ca

A. L. Martel: Email: anne.martel@sri.utoronto.ca 
of nuclei. Large connected components were separated using an automatic graph coloring method. ${ }^{4}$ Wienert's et. $a l^{2}$ approach was to first find an over segmented image of the slide consisting of all possible closed contours, they were then further evaluated and optimized to find and remove the contours that do not represent nuclei. Large contour areas (containing more than one nuclei) were then separated by eliminating the pixels at concave borders of objects and separated objects were further classified as either cell nuclei or other objects by putting a threshold on the Hematoxylin color channel of the image slide after separating the dye specific color channels using the method proposed by Ruifrok et al..$^{5}$

To the best of our knowledge there is only one study in the literature that tries to enhance the color uniformity of the image as a preprocessing stage to improve nuclei segmentation. Their idea was to use the prior knowledge of pathology dye colors to make the color distribution of the image uniform while keeping the edges of nuclei using a stain guided mean-shift filtering. ${ }^{6}$ This technique is iterative for each pixel vector and hence is lengthy to apply on large datasets. In this study, we introduce a color preprocessing method that considers the whole colorspace at once and hence is faster than an iterative process to apply on large images.

\section{METHODOLOGY}

Figure 1(a) shows a sample pathology slide available in the groundtruth dataset from the Wienert's et. $a l^{2}$ study. It is shown in Fig. 1(b) that the pixel values of this image are correlated in the red-green-blue (RGB) colorspace. This implies that if we want to find a threshold value which linearly separates blue/purple color (corresponding to Hematoxylin dye) we may include/exclude some unwanted/wanted pixels only because they are fairly close (correlated) to each other in this colorspace. Therefore, decorrelated and stretched colorspaces would enable us to treat each channel separately and each color independent from the others. ${ }^{7}$ This idea is shown in Fig. 1(c) after the RGB colorspace (in Fig. 1(b)) was decorrelated and stretched to fill the colorspace. Decorrelation Stretch is an image enhancement technique widely used in remote sensing to improve image quality of multi-spectral satellite imagery. Decorrelation stretching is done by rotating the RGB pixel values into a new space defined by the eigenvectors of the correlation matrix between the RGB bands, stretching the contrast of each color pixel with respect to the eigenvalues of the color correlation matrix from the previous step and then rotating the scaled values back into the original RGB space. ${ }^{8}$ The contrast stretching vector is calculated as:

$$
S=1 / \sqrt{e_{r g b}}
$$

where $S$ is a diagonal matrix containing the stretch factors, and $e_{r g b}$ is a diagonal matrix containing the eigenvalues of the three channels.

Next, the original RGB pixel values $x$, are transformed to a value in the new space $y$, using the uncorrelated and normalized matrix $S$ in the eigenspace:

$$
y=\left(E^{T} S E\right) x
$$

where $S$ is the diagonal stretching matrix, and $E$ is the eigenvectors of the correlation matrix of the RGB space. This essentially means to match between the mean and variance of the original RGB space to that defined by the eigenspace of the decorrelated and stretched target space. This technique was also used to enhance the H\&E stained images used in. ${ }^{9}$ Please note that the pixel colors in Fig. 1(c) correspond to the associated pixel values in Fig. 1(b) before decorrelation stretching.

After the original RGB image was transformed into a new image using the decorrelation stretching technique, it was converted from RGB to Lab colorspace and its gray scaled image was obtained using a weighted sum of the L, a, and b components as given in (3) (Fig. 2(b)).

$$
I_{\text {gray }}=0.25 * L+0.5 * a+0.25 * b
$$




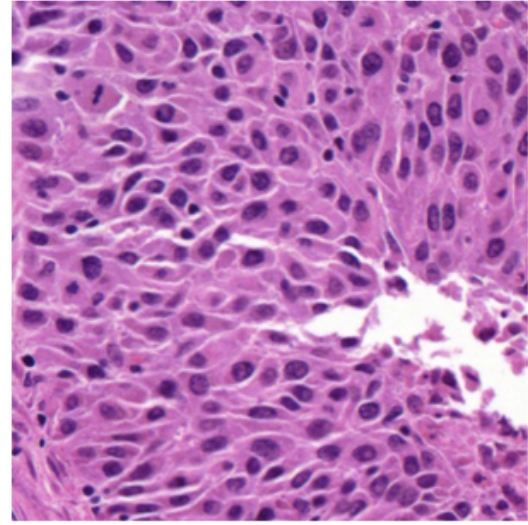

(a)

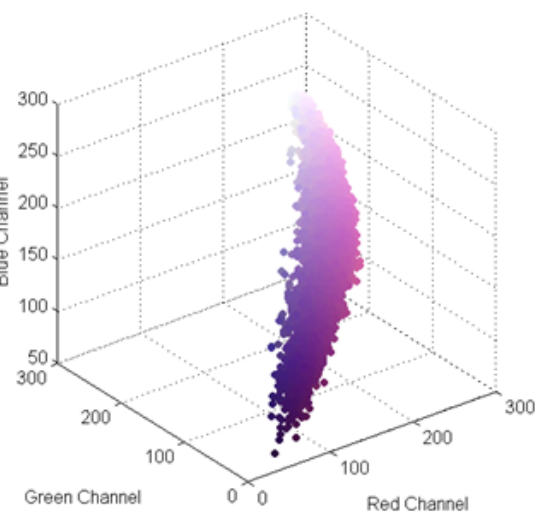

(b)

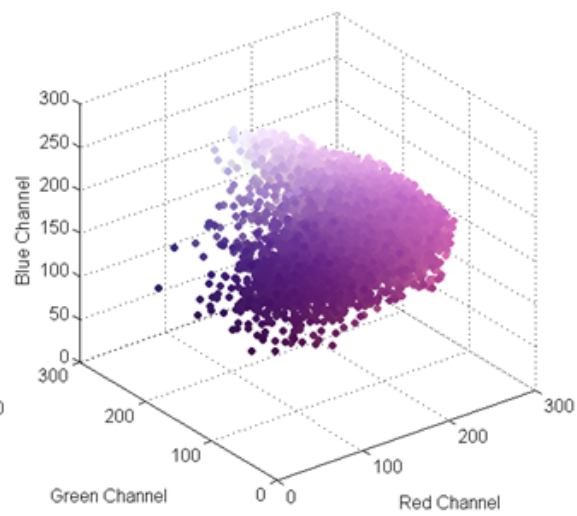

(c)

Figure 1. Result of applying decorrelation stretching on RGB colorspace of a pathology image patch. (a) original image, (b) original RGB colorspace, and (c) decorrelated and stretched new RGB colorspace.

This produces an image with foreground (nuclei) components to be darker than the background. Multilevel Otsu's thresholding followed by morphological operations were applied on the gray scaled image to find binary mask of the foreground nuclei regions. Large connected component blobs (area > 300 pixels) from the binary mask image are considered to enclose more than one nucleus. Therefore, they are divided into smaller regions by calculating the inverse binary distance transform of the blobs and generating markers from their centroids. Marker controlled watershed algorithm was then employed to find the location(s) at which the blobs are to be divided.

\section{RESULTS}

Showing an image from our in-house dataset motivates the need of a preprocessing step before attempting to detect/segment cells from pathology images. Figure 2(c) shows a cell detection result without an appropriate preprocessing stage and Fig. 2(d) shows the same image with detected cells after applying a preprocessing step. Please note that in Fig. 2(c) the lightly stained nuclei compared to their surrounding lymphocytes were not properly detected without a preprocessing step while after preprocessing the image using our proposed technique they were detected as shown in Fig. 2(d).

We also tested our method on a publicly available groundtruth dataset of $36 \mathrm{H} \& \mathrm{E}$ stained image crops ${ }^{2}$ with 7931 labeled cells. This dataset consists of cells from breast, liver, gastric mucosa, and bone marrow specimens. Like the method validation procedure in, ${ }^{2}$ we considered the detected nuclei touching the border to be false positives in this groundtruth dataset. Detected nuclei centers using our method were marked as true positives if their distances to the marked centers in the groundtruth set were less than or equal to $6 \mu m$. Given true positive (TP), false positive (FP), and false negative (FN) cell detection events, Table 1 compares the following statistical metrics from our proposed method with those presented in Wienert et. $a l^{2}$ study:

$$
\begin{aligned}
& \text { Precision }=\mathrm{TP} /(\mathrm{TP}+\mathrm{FP}) \\
& \text { Recall }=\mathrm{TP} /(\mathrm{TP}+\mathrm{FN}) \\
& \text { Dice coefficient }=2 \times \mathrm{TP} /(\# \text { of cells in groundtruth set }+\# \text { of detected cells }) \\
& \mathrm{F} 1 \text {-score }=2 \times \text { Precision } \times \text { Recall } /(\text { Precision }+ \text { Recall })
\end{aligned}
$$

As can be seen, our proposed technique outperforms the other segmentation methods with a F1-score of 0.901( \pm 0.03$)$. We found a statistically significant difference in the F1-score value of our method compared with others in Table 1 using a pairwise dependent two-tailed t-test method. It is worth mentioning that both F1scores and dice coefficients of our technique for individual groundtruth images were higher than 0.8 (Fig. 3). For examples of our automated cell detection and segmentation please refer to Fig. 2 and 4. 


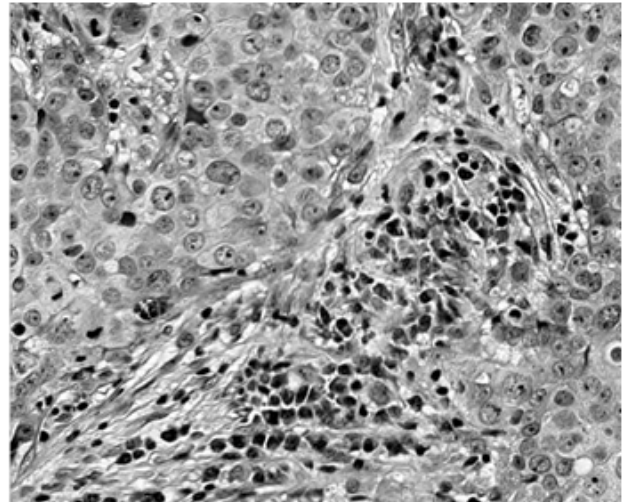

(a)

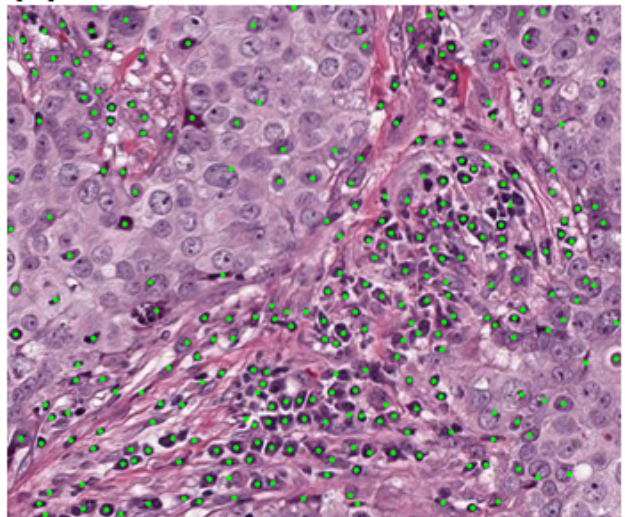

(c)

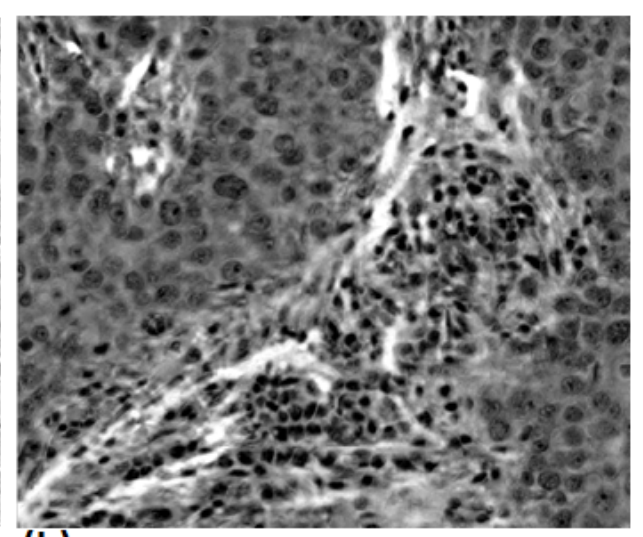

(b)

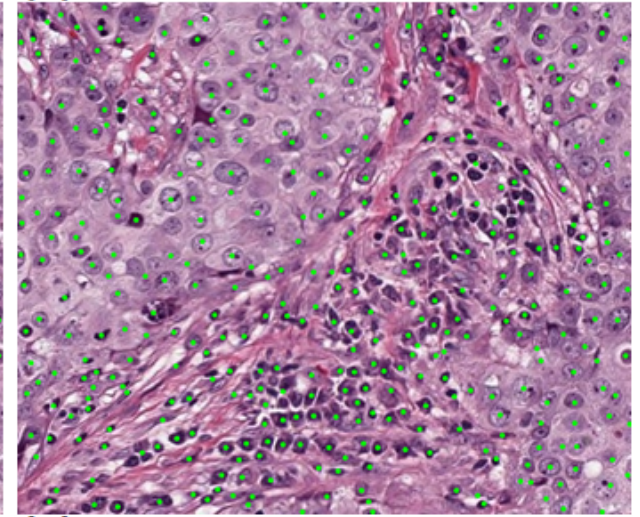

(d)

Figure 2. Effect of decorrelation stretching as a preprocessing stage on detecting cells from a pathology image patch. (a) gray scale image after original RGB image was converted to lab colorspace, (b) gray scale image after original RGB image was manipulated by decorrelation stretching and was converted to lab colorspace, (c) detected cells from gray scale image generated in (a) with no preprocessing stage, and (d) detected cells from gray scale image generated in (b) with decorrelation stretching as a preprocessing step.

Table 1. Performance summary of our proposed technique with those presented in Wienert et. al. ${ }^{2}\left({ }^{*}\right)$ indicates a statistically significant difference was found when a method was compared with our technique using a pairwise dependent two-tailed t-test method. The third row corresponds to performance of an optimized version of Al-Kofahi's method. Please note that the time performance for the first three methods are those as reported in ${ }^{2}$ while the time performance of our proposed method is based on the experiments done on a 64-bit Intel(R) Xeon(R) CPU (at 3.50 GHz) machine.

\begin{tabular}{cccccc}
\hline Method & Precision & Recall & Dice Coeficient & F1-score & Time (ms) \\
\hline \hline Wienert et. $a l^{2}$ & $0.908( \pm 0.05)$ & $0.857( \pm 0.04)$ & $0.821( \pm 0.05)$ & $0.881^{*}( \pm 0.03)$ & $725( \pm 184.9)$ \\
Al-Kofahi et. $a l^{3,10}$ & $0.707( \pm 0.14)$ & $\mathbf{0 . 9 1 6}( \pm \mathbf{0 . 0 4})$ & $0.761( \pm 0.09)$ & $0.789^{*}( \pm 0.09)$ & $679( \pm 52.3)$ \\
Al-Kofahi et. $a l^{3,10}$ & $0.811( \pm 0.10)$ & $0.913( \pm 0.04)$ & $0.820( \pm 0.06)$ & $0.854^{*}( \pm 0.06)$ & $1942( \pm 331.5)$ \\
Our method & $\mathbf{0 . 9 1 3}( \pm \mathbf{0 . 0 5})$ & $0.896( \pm 0.06)$ & $\mathbf{0 . 8 7 4}( \pm \mathbf{0 . 0 3})$ & $\mathbf{0 . 9 0 1}( \pm \mathbf{0 . 0 3})$ & $\mathbf{5 2 2}( \pm \mathbf{3 2 8 . 6})$ \\
\hline
\end{tabular}



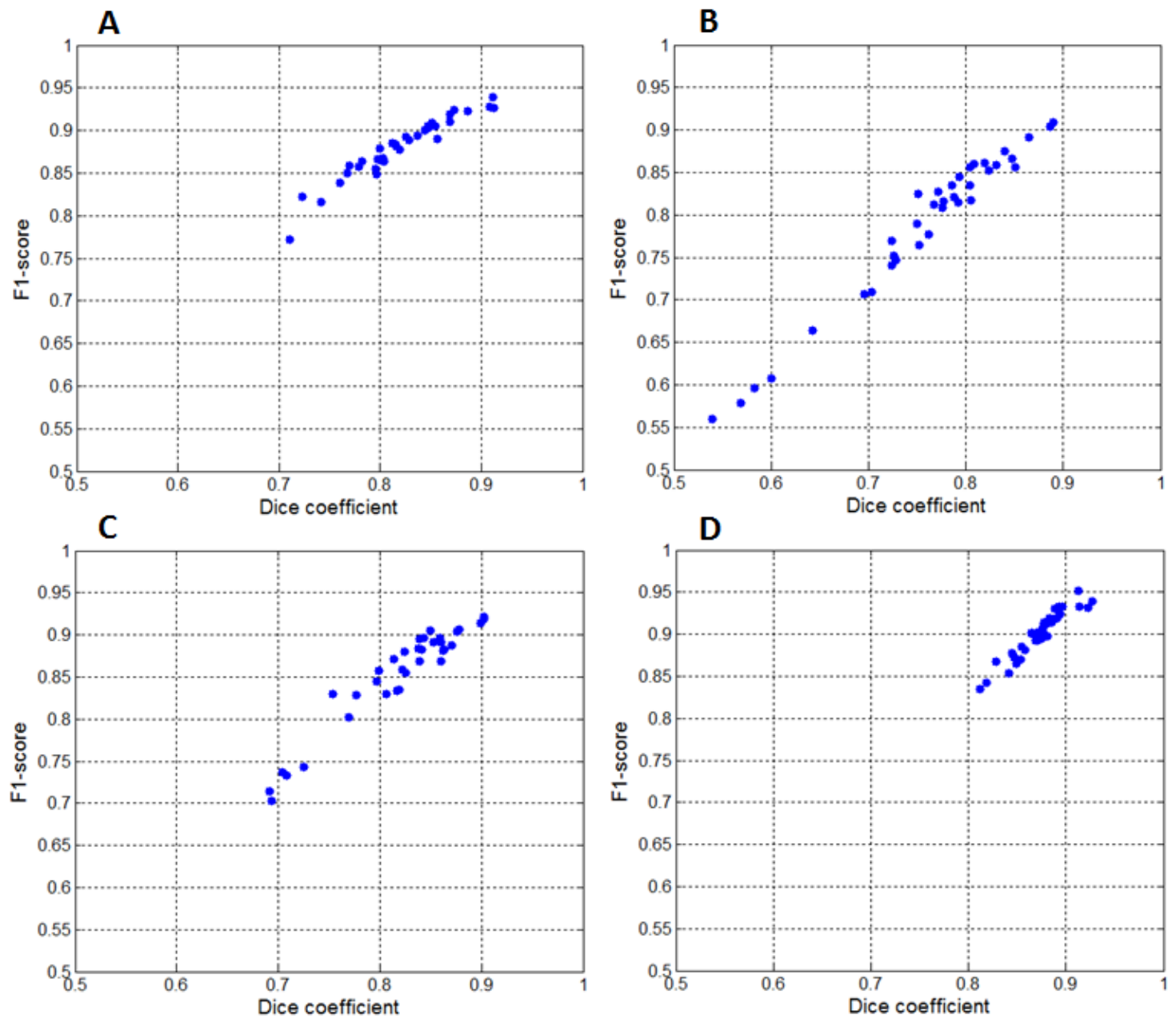

Figure 3. Performance measures corresponding to our proposed technique and different methods presented in Wienert et. $a l^{2}$ study. A) Wienert et. $a l,{ }^{2}$ B) Al-Kofahi et. $a l,{ }^{3}$ C) Al-Kofahi et. $a l,{ }^{10}$ and D) our proposed technique.
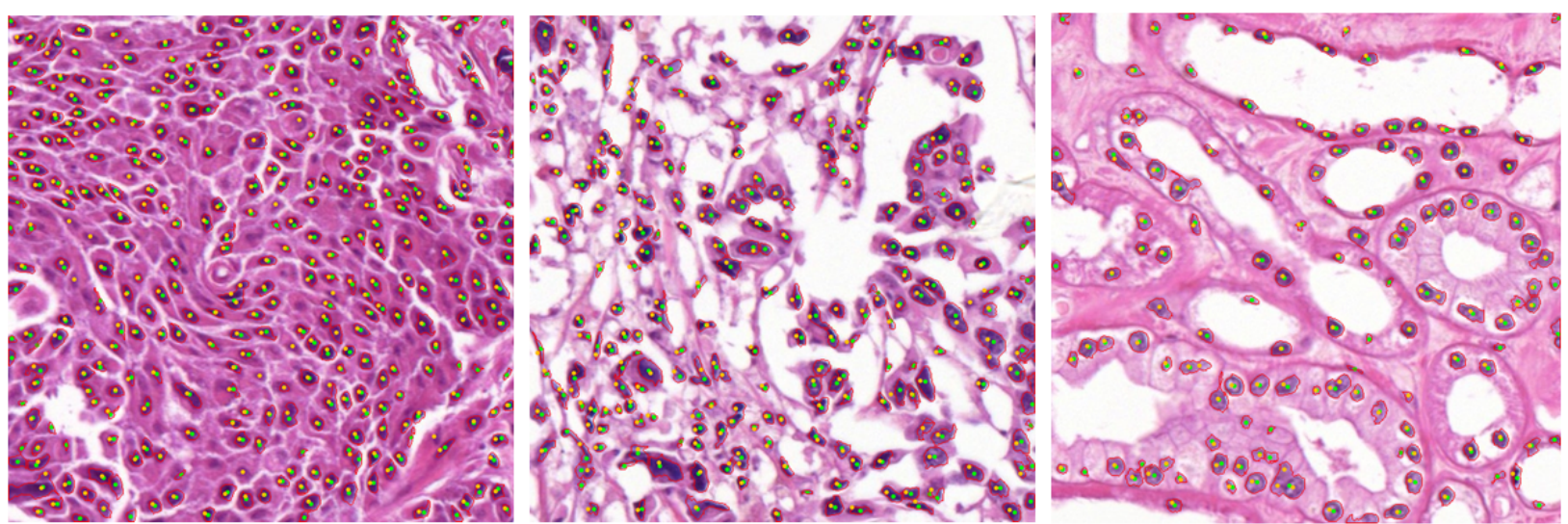

Figure 4. Segmentation results on different patches from validation set. Green dots indicate detection by our method, and yellow dots are the groundtruth manual detection by pathologists. 


\section{DISCUSSION AND CONCLUSIONS}

Most of the state-of-the-art cell detection and segmentation focus on complicated methods to subtract foreground cells from the background. In this study, we emphasize on the importance of preprocessing steps in having an improved and reliable segmentation.

We introduced a preprocessing technique to improve cell detection and segmentation from pathology images. This technique works on RGB colorspace to decorrelate and stretch pixel values of pathology images by transforming them into a new space defined by their top eigenvectors. Contrast for each pixel value was then stretched in this new space to equalize to the color variances, and transformed back to its original RGB space using the inverse transform. We have shown that this technique leads to a better segmentation performance when compared to other state-of-the-art methods. Although the application of this study was presented on cell segmentation, this technique may be helpful in other image processing applications where pixel values in color or gray scaled images are correlated.

\section{Acknowledgment}

This research is funded by the Canadian Cancer Society (grant number 703006).

\section{REFERENCES}

[1] Irshad, H., Roux, L., and Racoceanu, D., "Methods for Nuclei Detection, Segmentation , and Classification in Digital Histopathology : A Review Current Status and Future Potential," IEEE reviews in biomedical engineering 7, 97-114 (2014).

[2] Wienert, S., Heim, D., Saeger, K., Stenzinger, A., Beil, M., Hufnagl, P., Dietel, M., Denkert, C., and Klauschen, F., "Detection and segmentation of cell nuclei in virtual microscopy images: a minimum-model approach.," Scientific reports 2, 503 (Jan. 2012).

[3] Al-Kofahi, Y., Lassoued, W., Lee, W., and Roysam, B., "Improved automatic detection and segmentation of cell nuclei in histopathology images.," IEEE transactions on bio-medical engineering 57, 841-52 (Apr. 2010).

[4] Boykov, Y., Veksler, O., and Zabih, R., "Fast approximate energy minimization via graph cuts," Proceedings of the Seventh IEEE International Conference on Computer Vision, 377-384 vol.1 (1999).

[5] Ruifrok, A.C. \& Johnston, D., "Quantification of histochemical staining by color deconvolution," Analytical and quantitative cytology and histology, 291-299 (2001).

[6] Zhou, Y., Magee, D., Treanor, D., and Bulpitt, A., "Stain guided mean-shift filtering in automatic detection of human tissue nuclei.," Journal of pathology informatics 4, S6 (Jan. 2013).

[7] Reinhard, E., Ashikhmin, M., Gooch, B., and Shirley, P., "Color Transfer between Images," Computer Graphics and Applications, IEEE 21(5), 34-41 (2001).

[8] Gillespie, A. R., Kahle, A. B., and Walker, R. E., "Color Enhancement of Highly Correlated Images . I . Decorrelation and HSI Contrast Stretehe," Remote Sensing of Environment 20, 209-235 (1986).

[9] Belkacem-Boussaid, K., Samsi, S., Lozanski, G., and Gurcan, M., "Automatic detection of follicular regions in H\&E images using iterative shape index," Comput Med Imaging Graph 35, 592-602 (2011).

[10] Al-Kofahi, Y., Lassoued, W., Grama, K., Nath, S. K., Zhu, J., Oueslati, R., Feldman, M., Lee, W. M. F., and Roysam, B., "Cell-based quantification of molecular biomarkers in histopathology specimens," Histopathology 59(1), 40-54 (2011). 